\title{
Alterations in Body Weight, Blood Glucose Levels, and Lipid Profiles in High-Fat Diet-Low Dose Streptozotocin-Induced Diabetic Rats
}

\author{
Raysa Y. Pratiwi ${ }^{1}$, Berna Elya ${ }^{1, *}$, Heri Setiawan ${ }^{1}$, Atini Solawati ${ }^{1}$, Rosmalena $^{2}$
}

\section{Raysa Y. Pratiwi', Berna Elya ${ }^{1, *}$, Heri Setiawan' ${ }^{1}$, Atini Solawati ${ }^{1}$, Rosmalena ${ }^{2}$}

${ }^{1}$ Faculty of Pharmacy, Universitas Indonesia, Depok, INDONESIA.

${ }^{2}$ Faculty of Medicine, Universitas Indonesia Depok, INDONESIA.

\section{Correspondence}

\section{Berna Elya}

Faculty of Pharmacy, Universitas

Indonesia, Depok, INDONESIA.

E-mail: berna.elya@farmasi.ui.ac.id

History

- Submission Date: 02-09-2021;

- Review completed: 29-09-2021;

- Accepted Date: 06-10-2021.

DOI : 10.5530/pj.2021.13.199

Article Available online

http://www.phcogj.com/v13/i6

Copyright

(C) 2021 Phcogj.Com. This is an open access article distributed under the terms of the Creative Commons Attribution 4.0 International license.

\begin{abstract}
Introduction: New preventive and therapeutic strategies to treat Type 2 diabetes (T2D) continue to be pursued, the complexity of this disease makes it imperative to establish preclinical animal models which must provide accurate similarities to the pathogenesis of diabetes in humans. Making a diabetic animal model using rats with high-fat diet (HFD)-streptozotocin (STZ) induction is popular because it is relatively low cost and simple. Objectives: This study aims to analyse the changes in body weight, blood glucose, and lipid profiles that occur in diabetic rat models created by induction of HFD in combination with lowdose STZ. Methods: This study used forty male Sprague-Dawley rats (200-240 g). After the adaptation period, thirty rats were fed with HFD for 28 days (DM group), while the other ten rats continued to be fed with standard feed (NC group). After then, diabetes was induced to the DM group by low-dose STZ (35 $\mathrm{mg} / \mathrm{kg} \mathrm{BW}$ ). The body weight of the rats was measured before and after diet manipulation periods. Blood samples were taken before and after STZ induction to determine lipid profiles and blood glucose levels. Results: During the diet manipulation period, the HFD group experienced a significantly greater weight gain, higher blood glucose levels, and cholesterol (TC) levels. After STZ injection, rats' blood glucose levels, TC, and triglycerides significantly increased. Conclusion: HFD feeding combined with a low-dose STZ effectively work to mimic specific condition that is similar to T2D, and the stability of the experimental animal conditions remains constant for up to 6 weeks.

Key words: Diabetes, Insulin resistance, High-fat diet, Low-dose streptozotocin, Diabetic animal model, Stable diabetes type 2 profile.
\end{abstract}

\section{INTRODUCTION}

Diabetes can be interpreted as a metabolic disease which is characterized by high levels of glucose in the blood which can be caused by the body being unable to produce sufficient insulin or not being able to use it effectively. This disease is a longterm disease that will affect the quality of life. Uncontrolled diabetes will cause organ damage, especially to eyes, kidneys, nerves, heart, and blood vessels ${ }^{1}$. Both men and women are at risk for diabetes. There is no significant difference in the prevalence of diabetes at the age of 20-49 years between the two sexes ${ }^{2}$.

Diabetes is also known as a risk factor for COVID-19 disease. Patients with diabetes are twice likely to be put on a ventilator than patients who do not have diabetes. An epidemiological study has shown that diabetes increases the likelihood of critical illness and mortality in COVID-19 patients. A study in the UK involving 23,804 death cases due to COVID-19 reported that the probability of death increased up to 2-3 times greater in patients with diabetes ${ }^{3}$.

Diabetes has its own types, in which the types divided into Type 1 diabetes (T1D), and Type 2 diabetes (T2D). T1D is an autoimmune disease that causes $\beta$-cell damage. This disease can happen to all the age range, T1D commonly appears in childhood in their peak of puberty. Cases of T1D occur in both sexes in childhood, but in early adolescence, males are more likely to be diagnosed with the disease. While previously most common in Europe, now T1D is also becoming more common in other ethnic groups. The exact cause of T1D is still unknown, but it is clear that genetic and environmental factors play a role ${ }^{4}$. Whereas the signs on T2D, which is a complex metabolic disorder, can be characterized by altered lipid metabolism, insulin resistance, and pancreatic cell dysfunction. The particular factor that can lead to diabetes is obesity as for the development of $\mathrm{T} 2 \mathrm{D}$, which condition can lead to elevated triglycerides, hypertension, and insulin resistance ${ }^{5}$.

New preventive and therapeutic strategies to treat $\mathrm{T} 2 \mathrm{D}$ continue to be pursued, the complexity of this disease makes it imperative to establish preclinical animal models which must provide accurate similarities to the pathogenesis of diabetes in humans. Most animal models used for diabetes induction use chemicals that target pancreatic cells, for example, alloxan (ALX) and streptozotocin (STZ). STZ is known to be used for T1D or T2D induction. High doses of STZ will cause massive necrosis of $\beta$-cells, a condition similar to T1D. Aside from that, low doses of STZ can cause mild damage to $\beta$-cells and trigger an inflammatory process that is more likely to T2D condition ${ }^{5}$.

Unfortunately, animal modelling with low dose of STZ did not lead to insulin resistance, a condition commonly seen in T2D. Therefore, it is necessary to administer a high-fat diet (HFD) to animal models followed by low-dose STZ to mimic the pathogenesis of human T2D and not only in its phenotype. Giving HFD will trigger metabolic diseases such as hypertension, obesity, and the production of proinflammatory cytokines 5 . Based on these theories, this study aims to analyse the changes that occur 
in test animals induced by HFD-low-dose STZ and ensure that this induction is sufficient to create a stable and constant diabetic animal model for a certain time.

\section{MATERIALS AND METHODS}

\section{Materials and Preparation}

STZ was purchased from Wako, Fujifilm, Japan. Sodium citrate, citric acid, and other reagents were purchased from Sigma. HFD was made following Nascimento, et $\mathrm{al}^{6}$ and made in the commercial industry of animal feed manufacture (Indonesia Formula Feed, Indonesia). HFD contains $24 \%$ fat, $28 \%$ protein, and $33 \%$ carbohydrates.

\section{Experimental Animals}

Forty male Sprague-Dawley (SD) rats (8-12 weeks old, 200-240 g) were obtained from the National Agency of Drug and Food Control. Rats were acclimatized with free access to standard food ( $4 \%$ fat) and water for a week with the constant temperature which is $22 \pm 3^{\circ} \mathrm{C}$, humidity $(70 \pm 10 \%)$, and light-dark cycle $(12 \mathrm{~h} / 12 \mathrm{~h})$ at the Animal House, Health Science Cluster, Universitas Indonesia. This study was approved by the Ethics Committee of Faculty of Medicine, Universitas Indonesia (KET-868/UN2.F1/ETIK/PPM.00.02/2020) ${ }^{7}$.

\section{Induction of Diabetes}

After a one-week adaptation period, thirty rats were fed with HFD for 28 days (DM group), while the other ten rats continued to be fed with standard feed (NC group). After then, diabetes was induced to DM group by freshly prepared STZ (intraperitoneal). Freshly prepared STZ was made by dissolving STZ in $0.1 \mathrm{M}$ citrate buffer $(\mathrm{pH} 4.5,35 \mathrm{mg} / \mathrm{kg}$ $\mathrm{BW})^{5}$. Rats were fasted $4 \mathrm{~h}$ before STZ induction, and the feed was given immediately after injection. The DM group was still given HFD until the end of the study. Two weeks after STZ injection, rats in the DM group showing symptoms of polydipsia, polyuria, and fasting blood glucose (FBG) levels of $150 \mathrm{mg} / \mathrm{dl}$ were considered diabetic ${ }^{5}$.

\section{Measurement of Body Weight and Blood Glucose}

The body weight of the rats must to be measured before and after the process of diet manipulation periods. The weight gain of the rats in the DM group and the NC group was calculated and then compared. After the rats fasted for 9 hours, the process of measuring blood glucose in the rats was carried out ${ }^{8}$. Measurement of blood with a blood glucose monitoring device by first taking blood from the distal end of the tail which is then placed on a test strip for analysis (AccuChek Active, Roche Diagnostics, Mannheim, Germany).

\section{Lipid Profiles Analysis}

Blood samples were taken from the DM group before and after STZ induction. Blood samples were placed into plain tubes by retro-orbital sinus puncture and allowed to sit for one hour at room temperature to clot. Thereafter, serum collection and preparation by centrifugation of blood at $1000 \mathrm{x}$ g were carried out for ten minutes. Lipid profiles including triglycerides (TG), total cholesterol (TC), high-density lipoprotein (HDL), and low-density lipoprotein (LDL) were then measured with a DumoChem 20 (DUMO, Austria $)^{10}$.

\section{Statistical Analysis}

The data that presented fit as the mean \pm standard error of mean (SEM). Obtained data were analysed for normality and homogeneity of variance by the Shapiro-Wilk test and the Levene test, respectively. The student's t-test was used for analysing the data between two groups. Mean difference with a $\mathrm{p}<0.05$ value was considered significant. All statistical analyses were performed using GraphPad Prism version $8^{11}$.

\section{RESULTS AND DISCUSSION}

The number of percentages given by $\mathrm{T} 2 \mathrm{D}$ found in diabetes cases ranges from $90 \%-95 \%$. The highest average is found in countries with lower middle income. Thus, the case that occurs is an important concern for global health problems which can be influenced by various factors, namely irregular urbanization, extreme changes in the economy, culture and society, obesity, unhealthy lifestyles (both physical and food consumed), and too much in consuming foods that contain lots of sugar and or sweeteners. Diabetes can be treated by controlling the increase in blood sugar. According to World Health Organization, the criteria for establishing a diagnosis of diabetes are if clinical symptoms leading to diabetes accompanied by the results of a blood glucose test is the same to or more than $200 \mathrm{mg} / \mathrm{dl}$, fasting blood sugar equals to or more than $126 \mathrm{mg} / \mathrm{dl}$, plasma glucose 2 hours after meals equals to or more than $200 \mathrm{mg} / \mathrm{dl}$, or HbAlc equals to or more than $6.5 \%$ (48 $\mathrm{mmol} / \mathrm{mol})^{12}$.

People with T2D generally experience obesity which then triggers insulin resistance. Insulin is a hormone produced by pancreatic $\beta$-cells that facilitates the transfer of glucose to the body's cells, except for the brain, active muscles, and liver. When glucose absorption by cells is disturbed, glucose that cannot enter the cells will remain in the plasma, then increase the plasma glucose concentration. Several studies have shown that the responsiveness of skeletal muscle and liver to insulin can be modified by adipokines (hormones secreted by fat cells). Resistin can be released by fat which can interfere with insulin action by increasing insulin resistance. Increased production of resistin can occur in obesity, whereas adiponectin, another adipokine that can increase insulin sensitivity, decreases its production in obese conditions. In addition, an abnormal build-up of free fatty acids secreted from adipose tissue that occurs in muscle can result in impaired insulin action in muscle. Excess fatty acids can also indirectly trigger $\beta$-cell apoptosis. ${ }^{13}$

The administration of HFD in this study was expected to promote obesity in experimental animals to mimic obesity in human. As seen in Figure 1, HFD group experienced a significantly greater weight gain $(106.3 \pm 2.90 \mathrm{~g})$ compared to standard feed group $(64,93 \pm 3,27 \mathrm{gr})$. In addition, the HFD group also showed significantly higher RBG, FBG, and TC $\left(\mathrm{n}=6 ;{ }^{*} \mathrm{p}<0.05\right)$ (Table 1$)$.

The intervention required to cause obesity and progress to insulin resistance varies widely, ranging from 1 to 27 weeks. Obesity phenotype

Table 1: Blood glucose and cholesterol level of experimental animals between groups after 28 days diet manipulation $(n=6),{ }^{*} p<0.05$.

\begin{tabular}{|ccc|}
\hline Parameters $[\mathrm{mg} / \mathrm{dl}]$ & NC & DM \\
\hline RBG & $91.83 \pm 6.04$ & $111.8 \pm 3.24^{*}$ \\
FBG & $69.67 \pm 5.39$ & $85.00 \pm 3.18^{*}$ \\
TC & $62.67 \pm 3.90$ & $95.00 \pm 4.37^{*}$
\end{tabular}

RBG: Random blood glucose, FBG: Fasting blood glucose, TC: Total cholesterol.

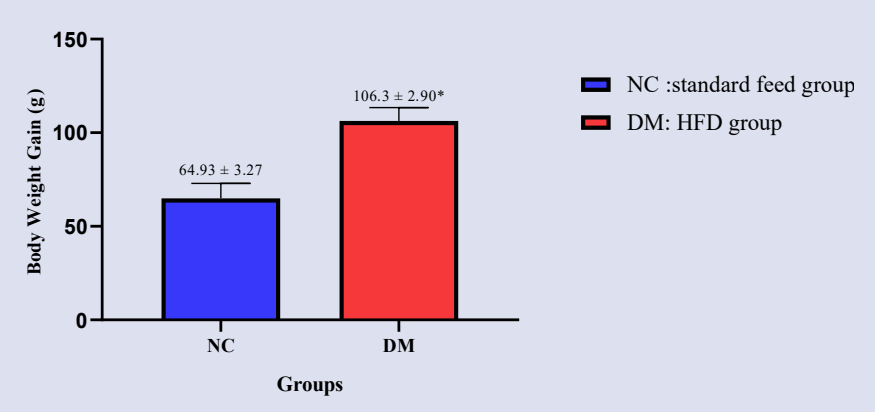

Figure 1: Body weight changes. Each point represents the Mean \pm SEM $(n=6)$, NC vs DM group * $p<0.05$. 
and metabolic changes will be more visible with the duration of the intervention. In this study, administration of HFD was carried out for 4 weeks, several phenotypes and metabolic changes of obesity began to be seen such as increased body weight, blood glucose, and cholesterol. Several studies have described several parameters of obesity, one of which is the presence of weight gain of more than or equals to $15 \%$ or $20 \mathrm{~g}$ in the test group compared to the control group. In this study, the weight gain of the HFD group met this parameter because the weight gain of the HFD group was more than $15 \%$ compared to the standard feed group. The increase in blood glucose also occurred. This might be because insulin metabolism cannot adapt to the damage caused by continuous excess calories caused by HFD. Also, HFD can induce fat accumulation in the pancreas which causes stress on $\beta$-cells and impairs insulin production. This can lead to hyperglycaemia. In the calculation of obesity, cholesterol is one of the important parameters which shows that the amount of serum cholesterol has a similar impact on fatty acid deposition in adipose tissue ${ }^{14}$.

Within four weeks of undergoing the stage of diet manipulation, rats in the DM group were given a low dose of STZ injection $(35 \mathrm{mg} / \mathrm{kg}$ BW) intraperitoneally. As seen in Figure 2, rats given STZ experienced a very significant increase in fasting blood glucose levels $(360.7 \pm$ $6.60 \mathrm{mg} / \mathrm{dl}$ ). This was because rats' $\beta$-cells were damaged due to the administration of STZ, causing a reduction in the insulin level, resulting hyperglycaemia. The diabetogenic effect of STZ is by causing damage to $\beta$-cells. GLUT2 is a plasma membrane-located glucose transporter that mediates the entry of STZ into $\beta$-cells and results in $\beta$-cells cytotoxicity. Among all the chemicals available to lead to diabetes, STZ is the most particular for modelling diabetes for the animals. STZ selectively damages $\beta$-cells resulting STZ to be unique and acceptable. Also, changes in physiological function and biochemistry found after STZ induction resemble those commonly found in diabetics. Therefore, the STZ-induced diabetes be a representative model that is considered medically relevant ${ }^{15}$.

Table 2: Lipid profiles of experimental animals before and after STZ injection ( $n=30)$. Before vs after, significantly different ${ }^{*} p<0.05$.

\begin{tabular}{|ccc|}
\hline $\begin{array}{c}\text { Lipid Profiles } \\
{[\mathrm{mg} / \mathrm{dl}]}\end{array}$ & Before & After \\
\hline TC & $96.5 \pm 5.53$ & $149.3 \pm 8.8^{*}$ \\
HDL & $60.72 \pm 2.03$ & $62.25 \pm 1.96$ \\
LDL & $66.17 \pm 2.66$ & $62.67 \pm 3.41$ \\
TG & $84.33 \pm 2.97$ & $488.3 \pm 65.08^{*}$ \\
\hline
\end{tabular}

TC: Total cholesterol, HDL: High density lipoprotein, LDL: Low density lipoprotein, TG: Triglyceride.

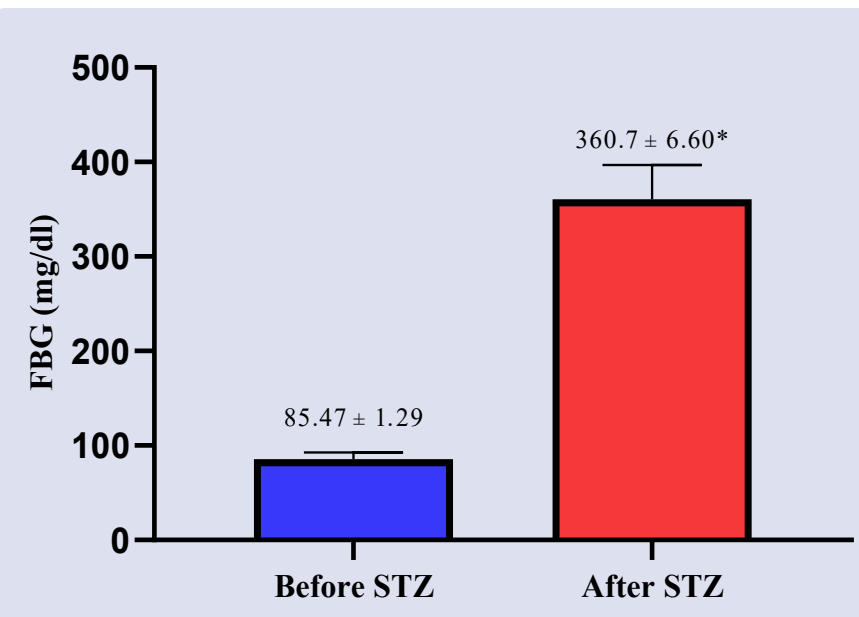

Figure 2: DM Group FBG changes. Data shown are means \pm SEM $(n=30)$. Before vs after, significantly different ${ }^{*} \mathrm{p}<0.05$.

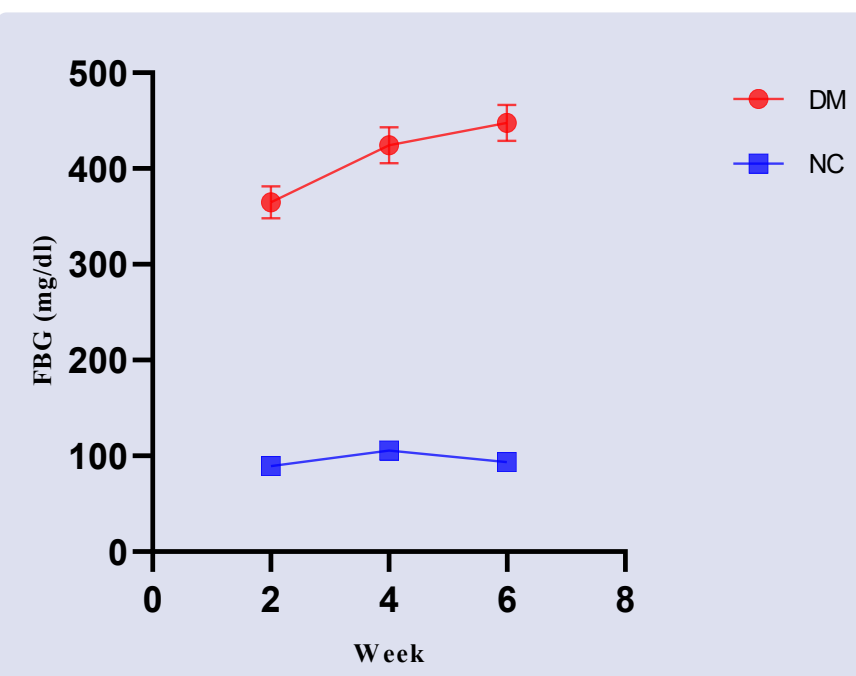

Figure 3: Fasting blood glucose level within 6 weeks. Data shown are means $\pm \operatorname{SEM}(n=6)$.

Administration of STZ also caused changes in the lipid profiles of animal models. Hyperlipidaemia is a complication of diabetes characterized by elevated levels of cholesterol and triglycerides. In this study, cholesterol and triglycerides in the DM group were seen to increase significantly (Table 3). While LDL and HDL remain unaffected. Insulin is called the "storage hormone" because one of its functions is to promote the storage of nutrients in the form of free fatty acids into the body's cells. Rats given STZ will experience $\beta$-cell damage which then leads to insulin deficiency conditions. Lack of insulin will result in high levels of free fatty acids in the blood, including cholesterol and triglycerides ${ }^{16}$.

STZ shows three glycaemic phases called "triphasic blood glucose" after STZ injection in response to destruction of $\beta$-cells. The initial hyperglycaemic phase is characterized by a decrease in insulin levels that can lead to a second hypoglycaemic phase due to the massive release of insulin from the ruptured $\beta$-cells. The last phase, the stable hyperglycaemic phase resulted in an increase in blood glucose levels up to $350-400 \mathrm{mg} / \mathrm{dl}$. This last phase occurs after approximately $1-2$ weeks after the STZ injection. Therefore, measurement of blood sugar after STZ induction should be done at least 2 weeks after injection ${ }^{15}$.

One of the factors that must be considered when making animal models is the stability of the animal model conditions during the study. Animal models are expected to survive under specific stable conditions for a certain period of time. In this study, clinical appearance and FBG of animal models were observed for 6 weeks after STZ injection. As seen in Figure 3, the FBG of the DM group remained in the diabetic state $(>150 \mathrm{mg} / \mathrm{dl})$. This shows that making animal models using HFDlow dose STZ is quite effective and efficient to make animal models that last up to 6 weeks ${ }^{11}$.

In addition to the HFD-STZ animal model, there are also genetically made diabetic animal models, for example Zucker diabetic fatty rat and $\mathrm{db} / \mathrm{db}$ mice. This animal model provides T2D conditions that are more similar than the HFD-STZ model, but the costs are very high, handling is difficult, and not always available. Other diabetic animal models, such as STZ-nicotinamide and neonatal STZ, require relatively large doses of STZ compared to HFD-STZ models and cause greater damage to cells. This animal model is more representative of T1D than T2D. Making a diabetic animal model using HFD-low dose STZ is preferred because it uses fewer doses of STZ, so costs can be relatively reduced, availability is maintained because it can use mice or rats, and based on this study, the HFD-low dose STZ animal model is quite effective to mimic T2D conditions within a specified timeframe ${ }^{5}$. 


\section{CONCLUSION}

The study focuses to investigate the changes of diabetic rats induced by HFD and low-dose STZ. From the data, HFD feeding combined with a low-dose STZ (35 mg/kg BW) injection effectively work to mimic specific condition that is similar to T2D for up to 6 weeks. However, further modifications of this model, such as increasing diet manipulation time, modifying the composition of HFD, or increasing study time, are still needed to investigate the stability and biochemical parameter changes of the animal models.

\section{CONFLICTS OF INTEREST}

The authors declare that they have no conflicts of interest.

\section{ACKNOWLEDGMENTS}

This research was supported by Universitas Indonesia through PUTI Grant 2020 [NKB-1456/UN2.RST/HKP.05.00/2020].

\section{REFERENCES}

1. IDF. IDF Diabetes Atlas 9th edition. The Lancet vol. 266 134-137 (2019).

2. Huebschmann, A. G., Huxley R. R., Kohrt, W. M., Zeitler, P., Regensteiner, J. G., Reusch, J. E. B. Sex differences in the burden of type 2 diabetes and cardiovascular risk across the life course. Diabetologia 62, 1761-1772 (2019).

3. Vas, P., Hopkins, D., Feher, M., Rubino, F. \& B. Whyte, M. Diabetes, obesity and COVID-19: A complex interplay. Diabetes, Obes. Metab. 22, 1892-1896 (2020)

4. Saberzadeh-Ardestani, B., Karamzadeh, R., Basiri, M., HajizadehSaffar, E., Farhadi, A., Shapiro, A. M. J., Tahamtani, Y., Baharvand, H. Type 1 diabetes mellitus: Cellular and molecular pathophysiology at a glance. Cell J. 20, 294-301 (2018).

5. Gheibi, S., Kashfi, K. \& Ghasemi, A. A practical guide for induction of type-2 diabetes in rat: Incorporating a high-fat diet and streptozotocin. Biomed. Pharmacother. 95, 605-613 (2017).

6. Nascimento, A. F., Sugizaki, M., Leopoldo, A., Lima-Leopoldo, A. P., Luvizotto, R. A. M., Nogueira, C. R., Cicogna A. C. A hypercaloric pellet-diet cycle induces obesity and co-morbidities in wistar rats. Arq. Bras. Endocrinol. Metabol. 52, 968-974 (2008).
7. Park, E., Lee, M. Y., Seo, C. S., Yoo, S. R., Jeon, W. Y., Shin H. K. Acute and subacute toxicity of an ethanolic extract of Melandrii Herba in $\mathrm{Crl}$ : CD sprague dawley rats and cytotoxicity of the extract in vitro. BMC Complement. Altern. Med. 16, 1-12 (2016).

8. Jensen, T. L., Kiersgaard, M. K., Sørensen, D. B. \& Mikkelsen, L. F. Fasting of mice: A review. Lab. Anim. 47, 225-240 (2013).

9. Wediasari, F., Nugroho, G. A., Fadhilah, Z., . Elya, B., Setiawan, H., Mozef, T. Hypoglycemic Effect of a Combined Andrographis paniculata and Caesalpinia sappan Extract in Streptozocin-Induced Diabetic Rats. Adv. Pharmacol. Pharm. Sci. 2020, (2020).

10. Chen, H. H., Chen, D., Chao, Y., Chen, Y., Wu, C., Lai, K. L., Lin, C H., Lin, C. C. Acarbose Decreases the Rheumatoid Arthritis Risk of Diabetic Patients and Attenuates the Incidence and Severity of Collagen-induced Arthritis in Mice. Sci. Rep. 5, 1-13 (2015).

11. Guo, X. xuan, Wang, Y., Wang, K., Ji, B. ping \& Zhou, F. Stability of a type 2 diabetes rat model induced by high-fat diet feeding with low-dose streptozotocin injection. J. Zhejiang Univ. Sci. B 19, 559-569 (2018).

12. WHO. Classification of diabetes mellitus 2019. Clinics in Laboratory Medicine vol. 21 1-13 (2019).

13. Bays, H., Mandarino, L. \& DeFronzo, R. A. Role of the Adipocyte, Free Fatty Acids, and Ectopic Fat in Pathogenesis of Type 2 Diabetes Mellitus: Peroxisomal Proliferator-Activated Receptor Agonists Provide a Rational Therapeutic Approach. J. Clin. Endocrinol. Metab. 89, 463-478 (2004)

14. de Moura e Dias, M., dos Reis, S. A., da Conceicao, L. L., de Oliveira Sediyama, C. M. N., Pereira, S. S., de Oliveira, L. L., do Carmo Gouveia Peluzio, M., Martinez, A., Milagro, F.I. Diet-induced obesity in animal models: points to consider and influence on metabolic markers. Diabetol. Metab. Syndr. 13, (2021).

15. Goyal, S. N., Reddy, N. M., Patil, K. R., Nakhate, K. T., Ojha S., Patil, C. R., Agrawal,Y.O. Challenges and issues with streptozotocininduced diabetes - A clinically relevant animal model to understand the diabetes pathogenesis and evaluate therapeutics. Chem. Biol. Interact. 244, 49-63 (2016).

16. Samuel, V.T. \& Shulman, G. I. The pathogenesis of insulin resistance: Integrating signaling pathways and substrate flux. J. Clin. Invest. 126 12-22 (2016). 


\section{GRAPHICAL ABSTRACT}

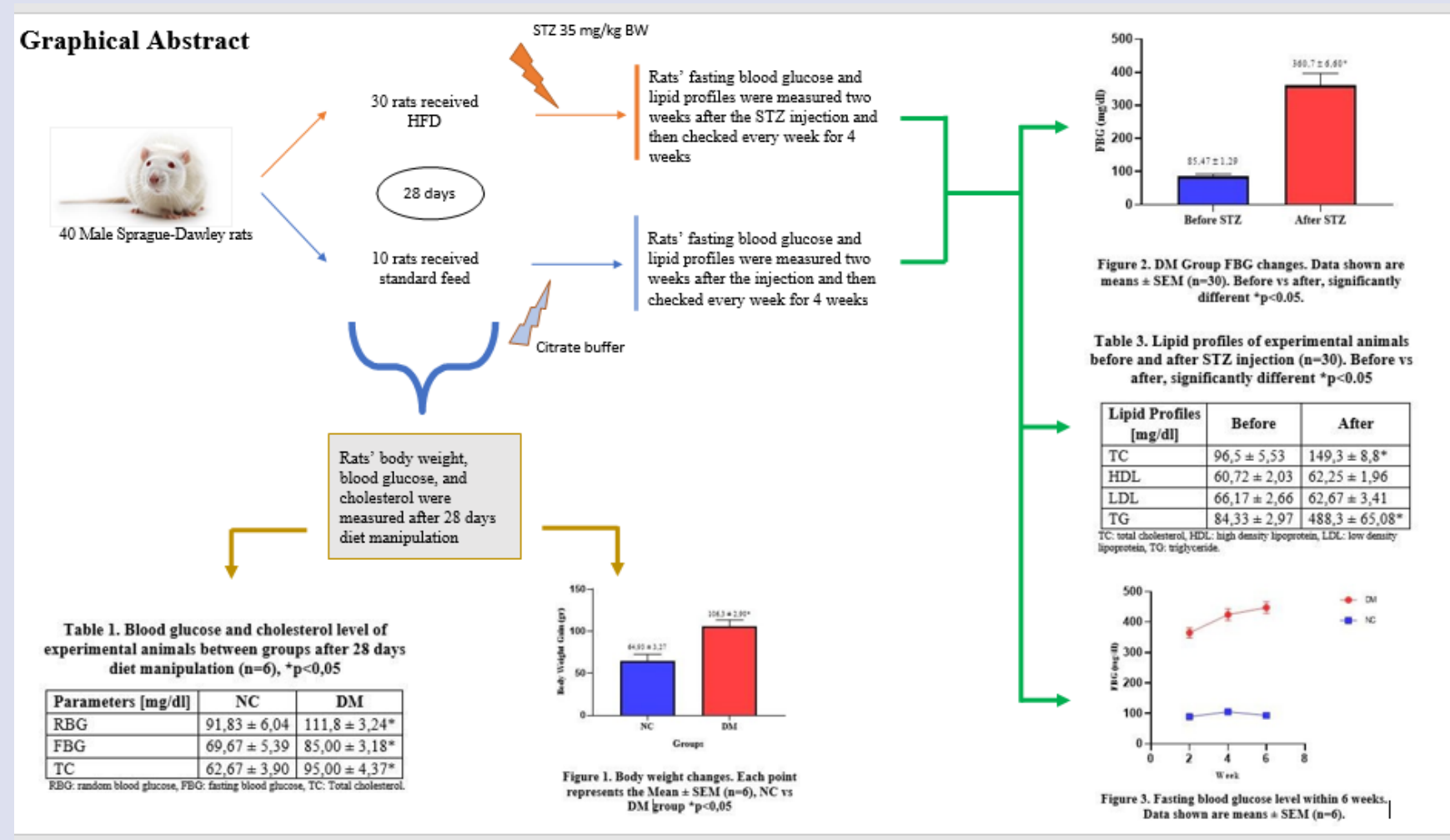

\section{ABOUT AUTHORS}

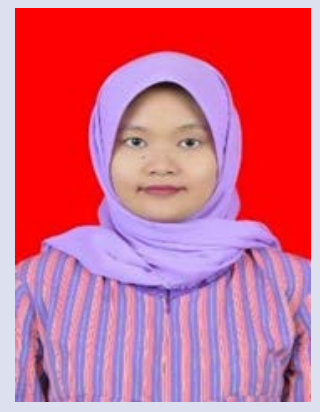

Raysa Y. Pratiwi is a Dentist and a Master of Herbal degree from Faculty of Pharmacy, Universitas Indonesia. Currently the research focuses on natural product, pharmacology and toxicology of herbal material.

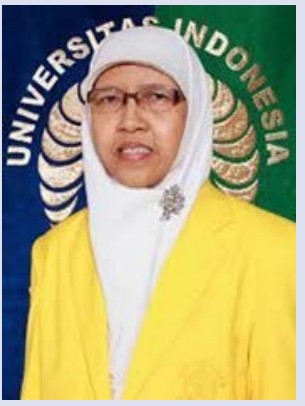

Berna Elya is a Professor and Lecturer at the Department of PharmacognosyPhytochemistry, Faculty of Pharmacy, University of Indonesia. She develops works in the field of pharmacognosy, phytochemistry, and natural material chemistry. 

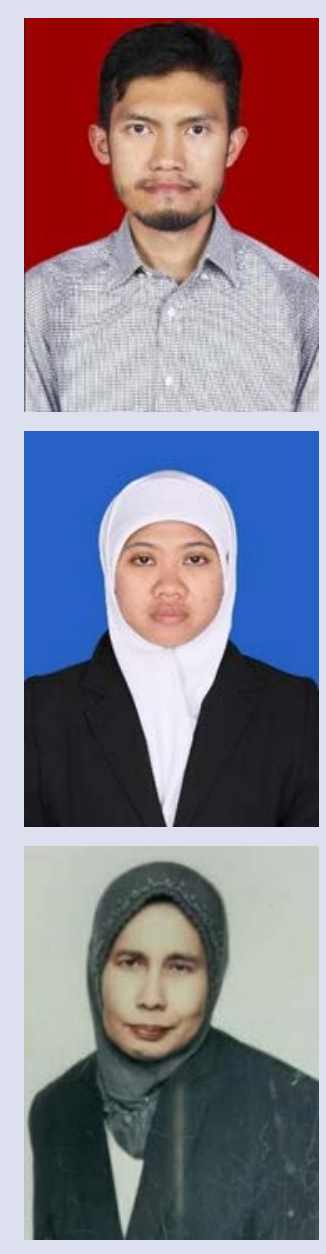

Heri Setiawan received Bachelor of Pharmacy from Universitas Indonesia in 2007, Master of Medical Science and Ph.D.in Medical Science from Okayama University in 2012 and 2016 respectively. He joined Faculty of Pharmacy Universitas Indonesia in 2019 as Lecturer in the field of Pharmacology and Toxicology. He is currently engaged in pharmacodynamics and toxicology study of compound to treat diabetes mellitus and gonadal hormones dysfunction.

Atini Solawati is a Veterinarian and a Master of Herbal degree from Faculty of Pharmacy, Universitas Indonesia. Currently the research focuses on herbal drug development, pharmacology and toxicology of herbal material.

Rosmalena is an organic chemist, Lecturer and Researcher at Medical Chemistry, Faculty of Medicine, Universitas Indonesia. Research interest natural product chemistry.

Cite this article: Pratiwi RY, Elya B, Setiawan H, Solawati A, Rosmalena. Alterations in Body Weight, Blood Glucose Levels, and Lipid Profiles in High-Fat Diet-Low Dose Streptozotocin-Induced Diabetic Rats. Pharmacogn J. 2021;13(6)Suppl: 1562-1567. 